\title{
Retroperitoneal Schwannoma: A Case Report
}

\author{
Dr.DhruvGupta ${ }^{1}$ Dr.DeepikaSharma ${ }^{2}$ Dr.KS Mehta ${ }^{3}$ Dr.Gurpreet ${ }^{4}$ Dr.AmitSuri ${ }^{5}$ \\ 1. Postgraduate Student Department Of Surgery ASCOMS And Hospitals Jammu (J\&K) India \\ 2.Postgraduate Student Department Of Surgery ASCOMS And Hospitals Jammu (J\&K) India. \\ 3.Professor Department Of Surgery ASCOMS And Hospitals Jammu (J\&K) India. \\ 4.AssistantProfessor Department Of Surgery ASCOMS And Hospitals Jammu (J\&K) India. \\ 5. AssociateProfessor (Urology) Department Of Surgery ASCOMS And Hospitals Jammu (J\&K) India.
}

\begin{abstract}
Retroperitonealschwannomais a veryrare primaryneurogenic tumour of the retroperitoneum with limited reporting of cases in the literature.It usually affects adults aged 20 to 50 yearsand has a male predominance.Symptomatology of schwannomas is highly nonspecific and dependson the location and size of the lesion. The majority of retroperitoneal schwannomas are benign in nature although malignant ones have also been reported. Its diagnosis and management poses a challenge for the radiologist and the surgeon.Authors are describing a case of "RETROPERITONEAL SCHWANNOMA" which wasreported in their centre.
\end{abstract}

Keywords: Retroperitoneal,Schwannoma, Neurogenic, Nonspecific, Challenge.

\section{Introduction:}

Schwannomas are slow growing solitary neural sheath tumors that originate from schwann cells. Commonly arising from cranial and peripheral nerves in the head and neck region or in the upper extremites[1]. However schwannomas may appear in the posterior mediastinum but rarely in the retroperitoneum, comprising $0.3 \%$ to $3.2 \%$ of allschwannomas[2]. In retroperitoneumthey can grow to a large size, sometimes displacing, and more rarely invading surrounding structures and organs before becoming clinical apparent.Presentation of the patient is often varied and sometimes its an incidental finding. Radiology can be used to make the diagnosis, to assess the extent of the lesion, tolook for features of malignant transformation but a correct preoperative diagnosisis is difficult to make and these tumors are often misdiagnosed.Tumour excision in toto is considered the treatment of choice.Confirmation of diagnosis is based on post operativehistopathologic examinationdemonstrating specific Antoni A/B areas and characteristic immunohistochemical features iepositive S-100 and a negative CD34[3].The prognosis of a benign schwannoma is good and recurrence is rare after complete excision.

\section{Case Presentation:}

Authors report a case of $69 \mathrm{yrs}$ old male patient resident of ranbirsinghpura,jammuretired army personelwho reported to our centrewithchief complaint of mild intensity painovercentral and right lower abdomen over a period of 3months. Pain was insidious in onset,non radiating, continuous and dull aching in character.Bowel and bladder habits were normal. No history of haemtemesis, malena, jaundice, prolonged feverand haemturia.Patient was a known diabetic and hypertensive for 6 years on medications.No similar pain complaints in the past nor any other family member had similar complaint. On clinical examination the patient was afebrile, pale and comfortable. Blood pressure and heart rate were normal. Abdominal examination was unremarkable with no abdominal mass palpable, no tendeness no hepatosplenomegaly with normal bowel sounds. Digital rectal examination was also normal. The chest X-ray, haemogram, biochemical analysis were all within the normal limits.Abdominal ultrasound and contrast enhanced CT scan revealed well defined heterogeneous contrast enhanced soft tissue density mass lesion measuring $12 \times 7 \times 5 \mathrm{~cm}$ at right paraspinal region with central necrosis[Fig $\mathbf{1} \mathbf{A}, \mathbf{B}]$. The mass was localized just above the right psoas muscle and inferior to the right kidney,slightly compressing the inferior vena cava. CT guided FNAC was planned but could not be done due to patient's disapproval. In view of above findings surgeons were confused about the type of neoplasm, about its malignant status and whether resectability would be possible due to its proximity to great vessels. A decision was reached that if the mass would be invading adjacent structures and intra operatively features suggested malignancy heroics of wide local excision would not be attempted and only biopsy specimen would be taken for histopathology and next step for management would be decided after histology report. On the contrary onsurgical exploration, a large encapsulated non invasive mass was found which was dissectible from the adjacent structures, it was very carefully dissected from inferior vena cava as it was almost impinging the large vein[FIG 2A,B,C,D]. It was possible to remove the complete tumour together with its capsular lining but no peripheral nerve entering or exiting the tumour was definable. Pathological and immunohistochemical 
evaluation revealed it to be a benign schwannoma (ancient variant). Postoperative and follow up period wasuneventful .

(FIG 1A, B) CECT ABDOMEN

(A)

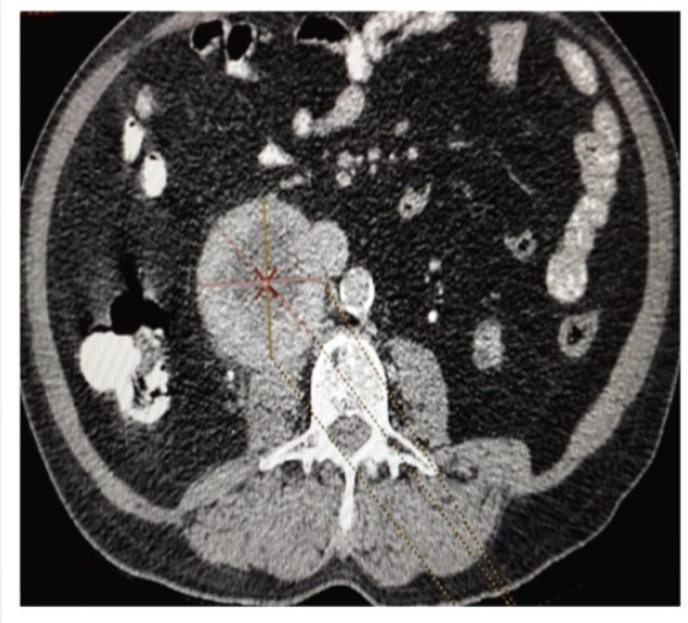

(FIG 2A,B,C,D) INTRAOPERATIVE IMAGES

(A)

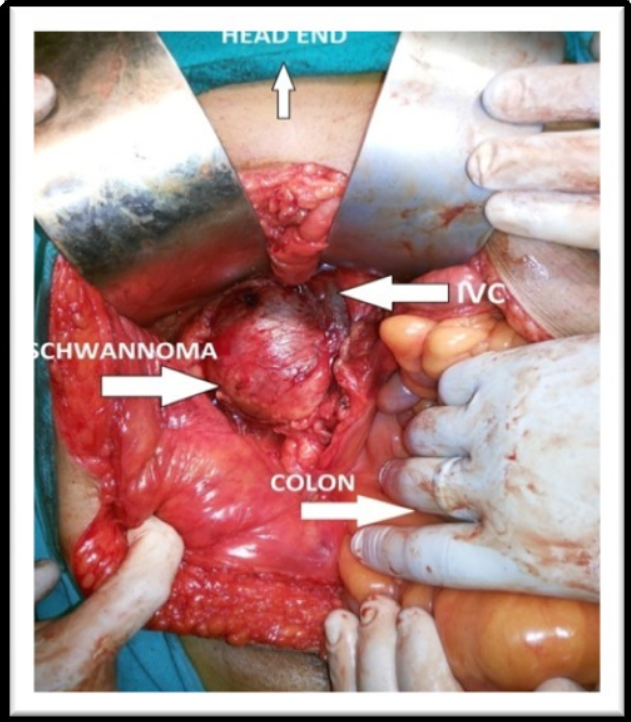

(C)

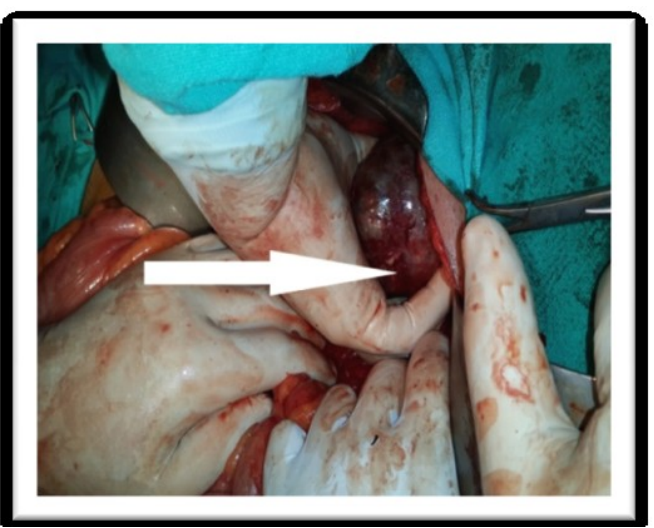

(B)

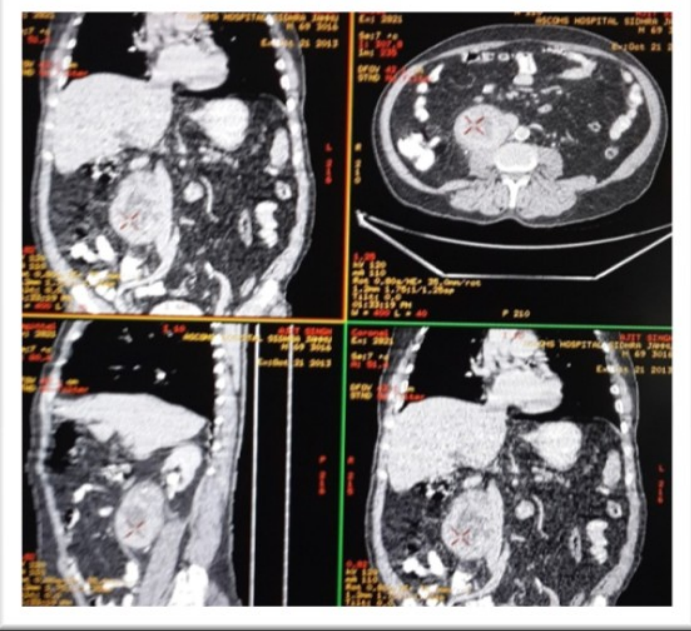

(B)

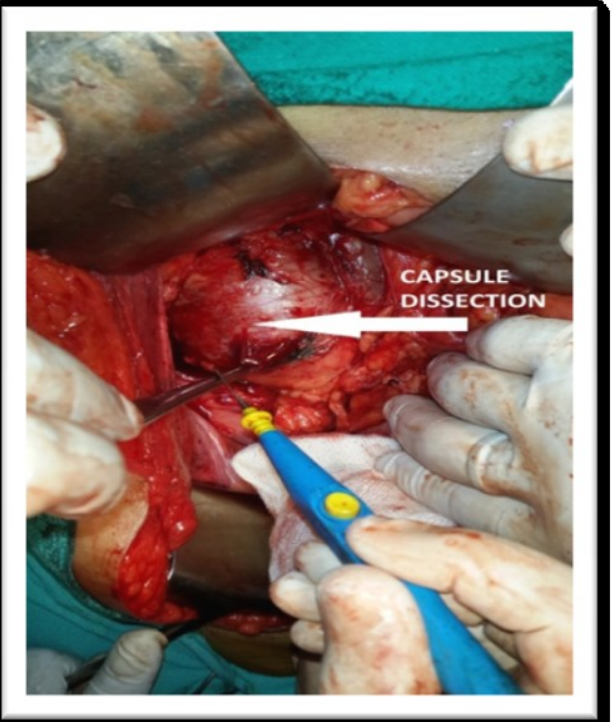

(D)

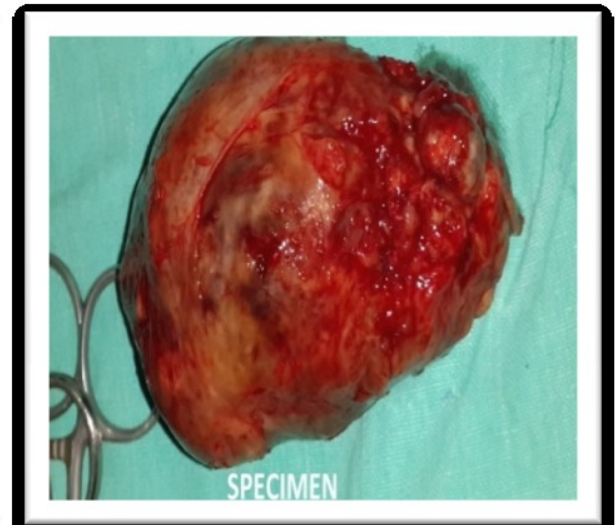




\section{Discussion:}

Schwannoma is a neurogenic tumor usually arising between the third and sixth decades of life, with a predilection for men. It may occur in any organ or nerve trunk with the exception of cranial nerves I and II which lack schwanncells. A schwannoma typically appears as a solitary, ovoid or spherical mass with welldefined borders, in large tumors $(>8-10 \mathrm{~cm})$ we may find degenerative pattern (cystic areas, calcifications, interstitial fibrosis, hyalinization) which is predominant in the "ancient" variant, a subtype of the typical or classic schwannoma with a very favorable clinical outcome. Early symptoms of retroperitoneal tumors are bizarre and none of the symptoms which occur can be considered diagnostic. Reported symptoms are vague, poorly localized abdominal pain and discomfort,accompanied by non-specific digestive disturbances. Referred pain and neurological symptoms in the lower extremities have also been described. The differential diagnosis for retroperitoneal schwannomasincludes other neurogenic tumors such as paraganglioma, pheochromocytoma ,liposarcoma and malignant fibrous histiocytoma.Hence the diagnosis of a benign retroperitoneal tumour is mainly one of exclusion, but ultimately based on histology.Therefore, misdiagnosis of retroperitoneal schwannomas is not uncommon.CT-guided core biopsy and fine needle aspiration have been founded to be unreliable for the diagnosis of retroperitoneal schwannoma[4]. They may be helpful only if thesample contains enough schwann cells to visualize microscopically.However, in areas of degeneration, the cellular pleomorphism can hinder the diagnosis, and degenerative cells may be misinterpreted as malignancy. One also runs the risk of hemorrhage, infection, and tumor seeding; thus, many authors do not recommend CT-guided biopsy. Therefore, surgical resection is the only accurate approach for pathologic evaluation to enable diagnosis of retroperitoneal schwannoma. Wide surgical resection in cases of benign retroperitoneal schwannomas has been advocated by some authorsbased on their belief that malignancy can never be totally excluded. Others believe that because this is a benign mass, a simple enucleation or partial excision of the tumor is sufficient . The argument here is that the morbidity associated with resection of adjacent tissue would not be justified in the treatment of a benign lesion[5]. Chemotherapy and radiotherapy have only limited role with marginal added benefit in malignant schwannomas[6]. The common pathological variants of schwannoma are (a) Conventional $\begin{array}{lllll}\text { schwannoma } & \text { (b)Ancientschwannoma } & \text { (c)Cellular schwannoma } & \text { (d)Plexiformschwannoma }\end{array}$ (e)Melanoticschwannoma and other rare variants[7]. Typical schwannomas are composed of inter mixed Antoni A components (cellular and arranged in short bundles or interlacing fascicles) and Antoni B areas (less cellular and organized with more myxoid components). Characteristically all schwannomas show uniform and intense staining for S 100 protein.

\section{Conclusion:}

The present case report emphasizes that surgeons should be aware of this rare neoplasm ie retroperitoneal schwannoma and its various non specific clinical associations and ways of presentations. Authors expressed the diagnostic and management dilemma which they faced and how they proceeded, perhaps this would aid other surgeons to manage such neoplasms in future.

DrDeepika Sharma

\section{Acknowledgments:}

PG Surgery ASCOMS

All financial support for the study was done by

DrDhruv Gupta and DrDeepikaSharma

\section{Ethical Approval Statement:}

Written informed consent was obtained from the patient for publication of this case report and accompanying images. A copy of the written consent is available for review by the Editor-in-Chief of this journal on request.

\section{References:}

[1]. Goh BKP, Tan Y, Chung YA, Chow PKH, Ooi LPJ, Wong WK. Retroperitoneal schwannoma. The American Journal of Surgery 2006;192:14-18

[2]. Melicow MM. Primary tumors of the retroperitoneum.Aclinicopathologic analysis of 162 cases: Review of the literatureand tables of classification. J IntCollSurg 1953; 19: 401-49.

[3]. Weiss SW, GoldblumJR.editors; Benign Tumorsof peripheral nerves.InEnzinger\& Weiss's soft tissue Tumors. Mosby, Messouri, USA, $200: 1146-1168$

[4]. Kishi Y, Kajiwara S, Seta S, Kawauchi N, Suzuki T, Sasaki K.Retroperitonealschwannoma misdiagnosed as a psoas abscess: report of case. Surg Today. 2002;32:849-52.

[5]. Gubbay AD, Moschilla G, Gray BN, Thompson I. Retroperitoneal schwannoma: a case series and review. Aust N J Surg 1995;65:197-200.

[6]. Shoji F, Maruyama R, Okamoto T, Wataya H, Nishiyama K, Ichinose Y. Malignant schwannoma of the upper mediastinum originating from the vagus nerve. World J SurgOncol 2005;3:65.

[7]. Kurtkaya-Yapicier O, Scheithauer B, Woodruff JM.The pathological spectrum of Schwannomas.HistolHistopath., 2003; 18(3): 925-934. 